\title{
Changing Social Learning Theory Through Reliance on the Internet of Things and Artificial Intelligence
}

\author{
Adam I. Attwood, $\mathrm{PhD}$ \\ Austin Peay State University, Clarksville, Tennessee, United States \\ (iD) https://orcid.org/0000-0002-4718-9272
}

Contact: attwooda@apsu.edu

\section{Abstract}

This essay examines the trend in the concepts of the Internet of Things (IoT), artificial intelligence (AI), and related computer-mediated teaching and learning. Implications for social learning theory are examined based on the concept of "cyborg" education through a variety of scholarly and popular media sources with implications for schools and teacher educators. Recommendations for teacher education are posited as the topic of technological mediation between people continues to change in relation to how education will likely need to adapt to provide a prosocial environment through technological mediation, though the social learning itself operates in different ways.

Keywords: social learning theory; virtual reality; artificial intelligence; cyborg; educational technology; Internet of Things

Date Submitted: April 25, 2020 | Date Published: June 15, 2020

\section{Recommended Citation}

Attwood, A. I. (2020). Changing social learning theory through reliance on the Internet of Things and artificial intelligence. Journal of Social Change, 12, 103-111. https://doi.org/10.5590/JOSC.2020.12.1.08

\section{Introduction}

Adolescent development is changing, as is the very concept of social engagement as more students in the $\mathrm{K}-12$ school system interact with nearly everything through screen mediation, interactive media, algorithm-driven marketing, and always-connected smartphones. According to Rogers' (2019) report, adolescents use computers for more than $7 \mathrm{hr}$ per day on average. O'Brien (2008) asked, "What if this fictitious use of technology was the norm?" (p. 383). Twelve years later, it is the norm. This statistic does not seem to be going down but is, instead, on an increasing trend (Fitzpatrick et al., 2020; Perry, 2017; Rogers, 2019), and this has accelerated in the year 2020 with enforced "social distancing" protocols that are changing society (see Lichfield, 2020). Social distancing protocols were discussed by Leeb et al. (2010) and have implications of legal and social importance as schools adapt.

The increasing reliance on automation and interactive technologies to increasingly mediate between people affects socialization skills and social dynamics between individuals and groups. Tally (2007) wrote alarmingly of this trend for education, asking critical questions that remained largely dormant and sidelined for more than a decade for several reasons, not least of which was the article's histrionic title perhaps ironically 
declaring "the end of social studies" that raised the proverbial specter of the Luddites. More specifically, the title suggested the end of social studies as it had been known before the full-scale integration of technology. What Burgess (2015) called "cyborg teaching" was accelerated by the advancement of the Internet of Things (IoT) that integrated artificial intelligence (AI). Schmelzer (2019) observed,

The Internet of Things is the collection of those various sensors, devices, and other technologies that aren't meant to directly interact with consumers, like phones or computers. Rather, IoT devices help provide information, control, and analytics to connect a world of hardware devices to each other and the greater internet. (para. 1)

Cyborg education, then, can be thought of as having three major components: (1) IoT, (2) AI, and (3) interconnected devices such as smartphones, smart watches, digital assistants that process multiple points of data semiautonomously or completely autonomously, and applications that can automate a process or activity.

Recently, Costa et al. (2019) raised a call for a new theorizing of educational technology for critical reflection during this time of increasing reliance on technology integration in schools that has, according to Statti and Torres (2020), generally been met with an uncritical attitude of inevitability. As Schaufele (2020) explained of questioning why students are reading certain texts in English language arts classrooms, a similar question could be asked of technology integration: Why is there more technology "inevitability" without questioning its social effects? Such a question would seem relevant for educator preparation programs to address as the trend toward cyborg education seems to be accelerating recently. This essay addresses what technology may mean for education going into the third decade of the 21st century and beyond for implications toward possibility and peril through a lens of social learning theory.

\section{Toward Cyborg Education Theory}

Schools have long been crucial in the social development of children and adolescents. With an increase in online learning without a physical classroom within which to congregate, social development itself is shifting. As this shift accelerated in 2020, education as a social enterprise continues to adapt both in its social mission as well as its content delivery and assessment of student learning outcomes. One of the ways to look at this shift is to view the increasingly technology-integrated school as part of what can be called cyborg education.

The word "cyborg" has origins in the 1960 (in Lexico, "Cyborg," n.d.-a; in Merriam-Webster, "Cyborg," n.d.b). Lexico defined cyborg is a person "whose physical abilities are extended beyond normal human limitations by mechanical elements built into the body." Merriam-Webster defined cyborg as simply "a bionic human." By those two definitions, an observer may reasonably say that human civilization is already comprised of cyborgs. A "smart" phone that most people use daily in industrialized and postindustrialized areas extends the physical abilities beyond normal human limitations to navigate, learn, engage in commerce, communicate in multiple languages with translation applications in real time and across continents, and to do many other activities.

Socialization is a major component of the importance of school, especially to foster prosocial behaviors. With an increasing emphasis on technology integration in schools, a question about the effects of such integration on student learning and socialization arises, even if such technology integration is mostly uncritically accepted (see Statti \& Torres, 2020). Deaton (2015) discussed some implications of online social media as to its effects on students' social development in schools. Deaton referenced Bandura's (1976) social learning theory to explore implications of online social media for student social development and noted that social learning theory itself is evolving as technological integration increases. Online social media has, according to Deaton (2015), "fundamentally changed the way that social interaction occurs within our world" (p. 4), and it is 
accessed through handheld devices such as smartphones, laptop computers, and in-ear Bluetooth devices by students on a regular basis that affects adolescent social development. Applying Bandura's social learning theory to this phenomenon of what can be called cyborg education indicates a mediating process of technology integration into and on the human body that conditions how adolescents interact with each other and those younger and older than them.

Popular media and scholarly sources alike have started to explore in more depth what used to seem like science fiction fantasy and is increasingly being seen as partial reality already with a sweeping momentum that seems to leave teachers little choice but to automatically accept cyborg education as the next chapter of the human condition (Alvarez, 2016; Bidshahri, 2018; Burgess; 2015; Gleason, 2014; Lombardo \& Blackwood, 2011; McPheeters, 2009; Perry; 2017). What has increasingly seemed "inevitable," however, is not a consistent trend, as it has had its awkward moments that have been criticized. Google Glass was an example of a "wearable" on the face that was heavily criticized and initially failed, but wearable technology gear has been reemerging, according to Wired Magazine among others (see Levy, 2017), with the successes of such wearable devices as the "smart" watch that integrates with a person's phone. Augmented virtual reality (VR) devices such as Google Cardboard have become increasingly used, so the initial failure of Google Glass may have been a temporary setback in the trend of wearable wireless technology (Dougherty, 2015; Levy, 2017).

VR has become increasingly mainstream for use in education (Cochrane, 2016). Merchant et al. (2015) noted that VR games such as Second Life could be used as a teaching tool in science subjects, including chemistry. Likewise, Gleason (2014) argued that the trend of cyborg-like teaching and learning should not be feared in science education but, instead, studied and understood for its potential to overcome what some have historically perceived as social inhibitors delaying entry into the highest levels of science industry.

If the student is increasingly integrated with technology, then so are the faculty. Burgess (2015) referred to faculty who teach online courses as engaging in "cyborg teaching" that could foster greater student learning than the nononline face-to-face format. It is futile, according to an opinion piece by Perry (2017), to resist this trend. Scholars, such as Gleason (2014), suggested an inevitability that should not be feared. The trend of increasing reliance on IoT has become embedded across society and, according to Schmelzer (2019), is becoming more sophisticated with AI. Nevertheless, Barlow-Jones and van der Westhuizen (2011) argued that the trend of technology integration needed to be examined critically to mitigate a divide in digital literacy based on access to resources. That said, they framed the issue of technology itself as neutral, while the implementation of resource allocation was the issue under examination. Digital literacy as a desirable quality was a given.

Digital literacy may be a given today as something to strive for across all population groups; however, the concept of cyborg education goes beyond literacy and is more of a social phenomenon in need of critical theorizing. McPheeters (2009) adapted Bandura's social learning theory to "cyborg learning theory" that blends the human mind with technology augmentation. In Quanta Magazine, Musser (2018) highlighted the potential of quantum computing to accelerate adaptive AI, or machine learning, which could result in a substantial expansion of human reliance on AI and the wearable (or even permanently embedded) machines that $\mathrm{AI}$ animates and controls on the human body. As schools increasingly integrate interactive, adaptive technology-and the AI that animates those tools-students may increasingly learn through AI mediation rather than through synchronous human interaction. An implication is the potentially substantial effect this may have on adolescent social development. As AI affects conditioning of human learning, global human demographics may be affected in new ways. The teacher could become decentered and asynchronous, while students learn from VR platforms controlled in part by adaptive AI. Although this can have positive benefits for students learning at different rates depending on cognitive ability and adjustable cognitive load for individual preferences, it can also have some unintended consequences for human social interaction that will modify adolescent behavior-and their later adult selves-in ways that may not be predictable. Culture is 
rarely static, but the conditioning effect of adaptive AI on culture will be a variable different than anything that came before it in pre-AI human conditioning.

If a cyborg future becomes more encompassing, then education will adapt. Adaptation of the school system may, however, not actually change the design of schools per se. Rather, schools might become more automated. Students will still go to a school building and to classrooms, but those buildings would be controlled by a potentially adaptive AI that wirelessly links to wearable devices or even embedded devices. Such a future is in small measure partially here already with smartphones, smart watches, Bluetooth earbuds, and interactive applications on phones and laptop computers. Mass media and scholarly observers are both addressing this apparent trend with some trepidation tempering an almost inexorable acknowledgement that technological mediation of human learning and interaction is here to stay permanently and will continue to evolve to be more, rather than less, present in daily life (Alvarez, 2016; Bidshahri, 2018; Burgess, 2015; Lombardo \& Blackwood, 2011; McPheeters, 2009; Perry, 2017).

Possibilities of cyborg education include its potential effect of increasing efficiency to foster student learning with differentiation. As more data is collected and algorithms and automated systems become more sophisticated, "Big Data" adds potential for supporting what could be considered more efficient teaching and learning and potentially more differentiated pacing of curriculum. In conjunction with AI and VR, the use of augmented reality (AR) can provide benefits for the online learning environment that overlays interactive virtual things into the physical world (Hampson, 2020). However, cyborg education also has potential for causing ambivalent changes to how people interact with each other (Fenwick \& Edwards, 2016). A pitfall of cyborg education is in its potential antisocial effects on adolescent social development (see Ma, 2011). If students interact more with AI-mediated computers than with human teachers and other students, what will likely result are changes in social development and, by extension, the application of social learning theory to school design. McPheeters (2009) called this "cyborg learning theory," in which teachers still control the AI's direction, override the AI, and can still always individually work with students. In other words, cyborg learning theory suggests that AI and machine learning in general, will never replace a human teacher. Rather, the machine integration makes learning more efficient allowing for teachers to have more time to teach students individually. The concept of cyborg education is recent and continually emergent as the technologies themselves have thus far been continually developing.

There is also a countermovement to reduce the amount of mediation that AI and wearable technology has on adolescents. For example, Sirin Kale (2018) interviewed teenagers who have opted out of online social media. Kale argued that the trend in technological mediation of human interaction is not inevitable, noting, "Amanda Lenhart, who researches young people's online lives, conducted a survey of U.S. teenagers ... found that $58 \%$ of teenagers said they had taken at least one break from at least one social media platform" (para. 18). Furthermore, "Of the young people Hill Holliday surveyed who had quit or considered quitting social media, $44 \%$ did so, she says, in order to "use time in more valuable ways"' (para. 19). However, "As young people increasingly reject social media, older generations increasingly embrace it: among the 45-plus age bracket, the proportion who value social media has increased from $23 \%$ to $28 \%$ in the past year, according to Ampere's data" (para. 4). The use of interactive, AI-mediated technology is not a clear or consistent trend among adolescents. Nevertheless, AI and wearable and embedded machines mediated by AI appears to be here to stay at every level of the education system. How AI is integrated in schools and how adaptive AI should be allowed to progress in the interconnected machines used in education will likely be an increasingly important open question that also seems to be pushing Bandura's social learning theory into a position of renewed importance this century. 


\section{Necessity of Technology Integration in Educator Preparation}

While the integration of technology in schools and curriculum at every level continues, there should be a critical evaluation of what technology is needed to foster learning. In addition to what technology is needed, teachers and administrators should critically evaluate how much technology should be used throughout the school day. Technology integration is embedded into the edTPA, which most teacher candidates must pass to be licensed to teach in the K-12 school system. For example, edTPA Task 2, "Instructing and Engaging Students in Learning," requires teacher candidates to implement technology into their lessons with the understanding that they will be evaluated on their effectiveness of technology integration (see Stanford Center for Assessment, Learning, and Equity, 2019). Likewise, the $\mathrm{C}_{3}$ Framework for social studies education emphasizes "college, career, and civic life" (National Council for the Social Studies, 2017). Civic life is the component that is immediately affected by technology integration in which students spend many hours a day on computers that mediate most communication and knowledge dissemination, students interact with wearable devices, and students seem to increasingly identify information through the algorithms of semiautonomous online programs. This is not necessarily "bad"-it may even be largely beneficial; however, questions should be asked, and implications should be evaluated. Implications for educator preparation programs include what should probably be a holistic revision of the educational technology curriculum across content areas so that preservice teachers can be more likely to proactively adapt to the possibilities and challenges of accelerated technology integration.

The necessity to integrate technology into $\mathrm{K}-12$ curriculum is in parallel with accountability measures to encourage the use of data-based decision-making, in which-according to Perry (2017) - "some studies in controlled, non-classroom environments reveal that typical students master content better when they handwrite notes compared to when they type" (para. 2). While the apparent inevitability of technology integration has largely gone unquestioned, the amount of integration has been contested in scholarly and popular media venues. Disagreement has tended to center around accommodations. According to Pryal and Jack (2017), students with disabilities need technology (also see Perry, 2017). Other scholars have taken a cautious approach to how much technology should be integrated and argued that it needs to be intentional for the given task. For example, using laptops during a lecture can be extremely distracting, according to Dynarski (2017). For Pryal and Jack (2017), the benefits outweigh the negatives.

When a person uses a smartphone or VR technology, they are augmenting their capabilities beyond the regular human ability to access, process, and interact with information, tools, and creativity. The smartphone is a computer that adds software and a hard drive to a human's processing power. Evolving technology and its implications for schools are part of the "futurist" discussion that McPheeters (2009) has suggested is necessary to address so that schools are more proactive rather than reactive to technological innovation and the changes that will potentially affect students. Likewise, Gleason (2014) suggested that educators should be proactive to address technology as a tool for change in education, because students themselves are driving part of that change. Instead of only trying to have students put away their phones or other wearable device, such as a smart watch, find ways to incorporate those technologies into the curriculum to achieve learning goals. This will result in some changes to the role of a teacher, but if teachers proactively address technological change, there can also be more of a role for teachers to take (Jukes et al., 2010). Perhaps technology itself is neutral, but all things do not remain equal as the ways in which different technologies change and interact throughout time is affected by students, teachers, administrators, policy makers, corporations, and other stakeholders. Proactively looking ahead is a recommendation to mitigate, if not avoid, the pitfalls of cyborg constructs of education some of which are especially challenging to even identify. Revisiting Bandura's (1976) social learning theory provides some insight, but now is the time to renew a critical evaluation of the trend toward cyborg education. An acceleration of this trend affects social learning theory for its implications in curriculum design and the school environment in general. 
As the interaction with ideas becomes increasingly filtered through the lens of computers, smartphones, and the increasingly sophisticated algorithms behind the Internet, student learning continues to be affected. These effects will have an impact on social learning. This trend began in 1991 with the emergence of the commercial Internet and computers being in most schools. According to an early report on technology integration in schools, Cuban (1992) noted that the use of computers in the real-time classroom "alters how teachers teach .. . and how students learn" (para. 12). This was generally seen as a positive phenomenon in that the integration of technology seemed to encourage teachers to "move from whole-class instruction to small groups and individualized options" (para. 12), though that is not always the most effective strategy to teach content. The evolution of computer technology has come a long way since 1992, but even now, nearly the same could be said about what seems likely and what could be unpredictable about integrating the next iteration of technological change in schools. Now, it is not computers per se that are the innovation but, rather, the advancing adaptive potential of VR (see Zimmerman, 2019) and, ultimately, AI (see Musser, 2018). VR in education is now essentially mainstream (Cochrane, 2016). However, Fitzpatrick et al. (2020) concluded in their study that "students who switched to virtual charter schools experienced large, negative effects on mathematics and English/language arts achievement that persisted over time." Findings such as this suggest the need to theorize cyborg social learning theory. Recent research studies such as the study conducted by Fitzpatrick et al. (2020) have raised questions about the efficacy of accelerating the integration of VR and automation technology in $\mathrm{K}-12$ education. Nevertheless, even Fitzpatrick et al. (2020) noted that the somewhat negative results of their study were ambiguous, if not ambivalent, noting: "It is also possible that discussing classroom characteristics in a study of virtual charter schools relfects out-of-date thinking, given the typical understanding of the term classroom" (p. 173). With VR being mainstream, the next step would seem to be AR and an increasingly sophisticated adaptive AI that is integrated into how students interact with and learn ideas, content, and ways of thinking. VR, AI, and AR are here to stay. As researchers study the effects of these technologies on academic achievement as more schools conduct distance education or remote (online) education, there should also be an increased focus on how these technologies affect social learning.

\section{Recommendations}

Because IoT is here to stay, its accelerated integration should be modulated toward learning outcomes that emphasize both the state $\mathrm{K}-12$ learning standards as well as the less measurable social development skills embedded into schools as social institutions. Fully online formats of school mediated through IoT should not lose sight of social skills development that has been important in $\mathrm{K}-12$ schooling. IoT is causing social change as people use smartphones and applications augmented with AI and algorithms (Schmelzer, 2019). Educator preparation programs should strategically embed educational technology coursework throughout the program's course of study rather than concentrate it into a single course, as has traditionally been done. In effect, the goal is to move beyond an educational technology framework and toward a cyborg learning framework that integrates the trend of everyone accessing school through computers, their phones, and the Internet. All these interactive tools are interconnected in some way and affected by algorithms, virtual processes, and AI. Though AI is relatively rudimentary, at present, the trend with advancing computer processing power indicates potentially exponential increases in the adaptive ability of AI (see Musser, 2018). VR is also becoming more widely used for education as it is connected to AI (Dougherty, 2015; Fitzpatrick et al., 2020). As this trend continues, more planning on social development should be included to prepare preservice teachers for an increased likelihood of being assigned to teach $\mathrm{K}-12$ classes within online/remote formats in which the Internet and its related systems-such as the learning management system integrated with any number of tools such as virtual tools, and semi-autonomous interactive tools-will be not just tools that affect the majority of your class but may be the entire learning environment.

In addition to the recommendation to embed educational technology as cyborg education throughout an educator preparation program instead of having only a stand-alone educational technology course, an 
educator preparation program should include ways to manage and mitigate stress associated with increased use of computer technology and screen time. Smith et al. (2015) noted that for online instructors, "Unmanaged stress can lead to serious declines in productivity, morale, and overall health" (p. 56) and that the "danger is that the symptoms may creep up, and soon, the recipient is not aware that he or she is experiencing them. It is similar to catching a cold" (p. 61). Educator preparation programs should include modules with the embedded educational technology components of the plan of study that systematically teach and remind students of how to be socially healthy at an individual and group level when their education is almost completely, if not completely, online and mediated by a computer screen, a phone screen, and the related systems of online school.

\section{Conclusion}

Educator preparation programs have long had educational technology courses as a required component. An educational technology course by itself, however, is increasingly insufficient for educator preparation. Technology integration will need to be more than separate tools and potential use of a tool here or a tool there and, instead, an integrated technology environment will likely be the next step in the evolution of how to prepare preservice teachers for the next iteration of schools. Rather than discussing technology integration, social studies teacher preparation programs will likely need to discuss integrated technology environments that increase the strategic and systematic use of wearable devices, handheld devices, and embedded devices that are interconnected with wireless technologies and the Internet. If one of the next technological changes is adaptive AI or adaptive AR, then interdisciplinary social studies teacher preparation programs will need to adapt as much as the $\mathrm{K}-12$ system.

\section{References}

Alvarez, R. (2016, June 6). Are cyborgs the inevitable next step in human evolution? The Science Explorer. http://thescienceexplorer.com/technology/are-cyborgs-inevitable-next-step-human-evolution

Bandura, A. (1976). Social learning theory. Prentice-Hall.

Barlow-Jones, G., \& van der Westhuizen, D. (2011). Situating the student: Factors contributing to success in an Information Technology course. Educational Studies, 37(3), 303-320. https://doi.org/10.1080/03055698.2010.506329

Bidshahri, R. (2018, December 21). Educating the wise cyborgs of the future. SingularityHub. https://singularityhub.com/2018/12/21/educating-the-wise-cyborgs-of-the-future/

Burgess, O. (2015). Cyborg teaching: The transferable benefits of teaching online for the face-to-face classroom. MERLOT Journal of Online Learning and Teaching, 11(1), 136-144. https://jolt.merlot.org/vol11no1/Burgess 0315.pdf

Cochrane, T. (2016). Mobile VR in education: From the fringe to the mainstream. International Journal of Mobile and Blended Learning, 8(4), 44-60. https://doi.org/10.4018/IJMBL.2016100104

Costa, C., Hammond, M., \& Younie, S. (2019). Theorising technology in education: An introduction. Technology, Pedagogy and Education, 28(4), 395-399. https://doi.org/10.1080/1475939X.2019.1660089

Cuban, L. (1992, November 11). Computers meet classroom; classroom wins. Education Week. https://www.edweek.org/ew/articles/1992/11/11/10cuban.h12.html

Cyborg. (n.d.-a). In Lexico. https://www.lexico.com/en/definition/cyborg 
Cyborg. (n.d.-b). In Merriam-Webster.com dictionary. Retrieved March 7, 2020, from https://www.merriam-webster.com/dictionary/cyborg

Deaton, S. (2015). Social learning theory in the age of social media: Implications for educational practitioners. Journal of Educational Technology, 12(1), 1-6. https://files.eric.ed.gov/fulltext/EJ1098574.pdf

Dougherty, C. (2015, May 28). Google intensifies focus on its cardboard virtual reality device. New York Times. https://nyti.ms/1SEGYdH

Dynarski, S. (2017, November 22). Laptops are great, but not during a lecture or a meeting. New York Times. https://nyti.ms/2hVxlzm

Fenwick, T., \& Edwards, R. (2016). Exploring the impact of digital technologies on professional responsibilities and education. European Educational Research Journal, 15(1), 117-131. https://doi.org/10.1177/1474904115608387

Fitzpatrick, B. R., Berends, M., Ferrare, J. J., \& Waddington, R. J. (2020). Virtual illusion: Comparing student achievement and teacher and classroom characteristics in online and brick-and-mortar charter schools. Educational Researcher. 49(3), 161-175. https://doi.org/10.3102/0013189X20909814

Gleason, S. C. (2014). Don't fear the cyborg: Toward embracing posthuman and feminist cyborg discourses in teacher education and educational technology research. Canadian Journal of Science, Mathematics and Technology Education, 14, 120-134. https://doi.org/10.1080/14926156.2014.903320

Hampson, M. (2020, April 9). Mirror arrays make augmented reality more realistic. IEEE Spectrum: Technology, Engineering, and Science News. https://spectrum.ieee.org/techtalk/sensors/imagers/mirrors-augmented-reality-more-realistic

Jukes, I., McCain, T., \& Crockett, L. (2010). Education and the role of the educator in the future. Phi Delta Kappan, 92(4), 15-21. https://doi.org/10.1177\%2Fo03172171009200403

Kale, S. (2018, August 29). Logged off: Meet the teens who refuse to use social media. The Guardian. https://www.theguardian.com/society/2018/aug/29/teens-desert-social-media

Leeb, K., Chrysler, D., \& Goodman, R. A. (2010). The social distancing law project template: A method for jurisdictions to assess understanding of relevant legal authorities. Disaster Medicine and Public Health Preparedness, 4(1), 74-80. https://doi.org/10.1017/S1935789300002457

Levy, S. (2017, July 18). Google Glass 2.0 is a startling second act. Wired Magazine. https://www.wired.com/story/google-glass-2-is-here/

Lichfield, G. (2020, March 17). We're not going back to normal: Social distancing is here to stay for much more than a few weeks-It will upend our way of life, in some ways forever. MIT Technology Review. https://www.technologyreview.com/s/615370/coronavirus-pandemic-social-distancing-18-months/

Lombardo, T., \& Blackwood, R. T. (2011). Educating the wise cyborg of the future. On the Horizon, 19(2), 8596. https://doi.org/10.1108/10748121111138281

Ma, H. K. (2011). Internet addiction and antisocial internet behavior of adolescents. Scientific World Journal, 11, 2187-2196. https://doi.org/10.1100\%2F2011\%2F308631

McPheeters, D. (2009). Cyborg learning theory: Technology in education and the blurring of boundaries. World Futures Review, 1(6), 34-39. https://doi.org/10.1177/194675670900100605

Merchant, Z., Keeney-Kennicutt, W., \& Goetz, E. (2015). Predicting undergraduate students' acceptance of Second Life for teaching chemistry. MERLOT Journal of Online Learning and Teaching, 11(2), 233248. https://jolt.merlot.org/Vol11no2/Merchant 0615.pdf 
Musser, G. (2018, January 29). Job one for quantum computers: Boost artificial intelligence. Quanta Magazine. https://www.quantamagazine.org/job-one-for-quantum-computers-boost-artificialintelligence-20180129/

National Council for the Social Studies. (2017). The college, career, and civic life (C3) framework for social studies state standards: Guidance for enhancing the rigor of K-12 civics, economics, geography, and history [Report]. https://www.socialstudies.org/sites/default/files/2017/Jun/c3-framework-forsocial-studies-rev0617.pdf

O'Brien, J. (2008). Technology: An integral part of students' learning and lives. Social Education, 72(7), $383-385$.

Perry, D. M. (2017, December 6). The futile resistance against classroom tech. The Atlantic. https://www.theatlantic.com/education/archive/2017/12/laptops-in-the-classroom/547607/

Pryal, K. R. G., \& Jack, J. (2017, November 27). When you talk about banning laptops, you through disabled students under the bus. HuffPost. https://www.huffpost.com/entry/when-you-talk-about-banninglaptops-you-throw-disabled_b_5a1ccb4ee4bo7bcab2c6997d?ncid=engmodushpmg00000003

Rogers, K. (2019, October 29). U.S. teens use screens more than seven hours a day on average-and that's not including school work. CNN. https://www.cnn.com/2019/10/29/health/common-sense-kids-mediause-report-wellness/index.html

Schaufele, M. (2020). Why are we reading this? Hermeneutic inquiry into the practice of teaching (with) literature. Educational Studies, 56(2), 145-159. https://doi.org/10.1080/00131946.2019.1695212

Schmelzer, R. (2019, October 1). Making the Internet of Things (IoT) more intelligent with AI. Forbes. https://www.forbes.com/sites/cognitiveworld/2019/10/o1/making-the-internet-of-things-iot-moreintelligent-with-ai/\#5aobdfocfdgb

Smith, G. S., Brashen, H. M., Minor, M. A., \& Anthony, P. J. (2015). Stress: The insidious leveler of good, unsuspecting, online instructors of higher education. Journal of Social Change, 7(1), 56-68. https://scholarworks.waldenu.edu/jsc/vol7/iss1/5/

Stanford Center for Assessment, Learning, and Equity. (2019). Making good choices: Candidate support resource [Handbook]. https://www.edtpa.com/Content/Docs/edTPAMGC.pdf

Statti, A., \& Torres, K. M. (2020). Digital literacy: The need for technology integration and its impact on learning and engagement in community school environments. Peabody Journal of Education, 95(1), 90-100. https://doi.org/10.1080/0161956X.2019.1702426

Tally, B. (2007). Digital technology and the end of social studies education. Theory and Research in Social Education, 35(2), 305-321. https://doi.org/10.1080/00933104.2007.10473337

Zimmerman, E. (2019, August 22). AR/VR in K-12: Schools use immersive technology for assistive learning. EdTech Magazine. https://edtechmagazine.com/k12/article/2019/08/arvr-k-12-schools-useimmersive-technology-assistive-learning-perfcon

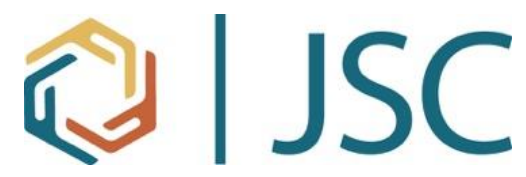

The Journal of Social Change, sponsored by Walden University, welcomes manuscripts focusing on interdisciplinary research in social change that improves the human condition and moves people, groups, organizations, cultures, and society toward a more positive future. 